\title{
EPILOGUE
}

\section{Renal cell carcinoma: currently the most interesting genitourinary malignancy}

\section{Lori A. Wood, MD, MSc (Epi), FRCPC; ${ }^{*}$ \\ Michael A.S. Jewett, MD, FRCSC, FACS ${ }^{\dagger}$}

From the *Department of Medicine, Dalhousie University, Halifax, NS, and the tDivision of Urology, and the Departments of Surgery and Surgical Oncology, Princess Margaret Hospital and the University Health Network, and the University of Toronto, Toronto, Ont.
A s can be seen from the preceding articles in this first supplement to the Canadian Urological Association Journal, major scientific and clinical advancements have been made in the molecular characterization and management of renal cell carcinoma (RCC). Radical nephrectomy for RCC was standardized by Robson and others ${ }^{1}$ in Toronto over 40 years ago, and laparoscopic techniques began with Clayman ${ }^{2}$ over 15 years ago. Partial nephrectomy, previously condemned, seems to provide equivalent cancer control in selected cases. ${ }^{3}$ These advances, coupled with improved imaging, were the major advances that had an impact on survival until the advent of new targeted therapies. Patients who developed metastatic disease were, at most, palliated by systemic therapies. This may be changing. The next decade will prove even more exciting for all those involved in the field of RCC as new local and systemic therapy advances are developed and build on the discoveries of basic science.

Questions in many areas remain unanswered. Better prognostic factors are needed to identify those patients with early-stage small tumours (now more than $50 \%$ of new cases) who could initially undergo active surveillance, delaying the need for therapy for progression of the disease. Similarly, patients with indolent metastatic disease may be better served by delayed treatment. Better definition of these patients through molecular or imaging characterization of their tumours would be a major clinical breakthrough.

New, less invasive therapies with radiofrequency ablation, cryotherapy and highintensity focused ultrasound are being used, but their long-term results are unknown, and more such therapies are under development. Laparoscopic partial nephrectomy is gradually being adopted as surgeons are trained to do this very difficult procedure.

Another issue arising from the explosion of these new systemic therapies is identifying which are superior for progression-free survival, overall survival and quality of life. With the array of potential therapeutic agents - interleukin-2, interferon- $\alpha$, sunitinib, sorafenib, temsirolimus, bevacizumab, RAD001 and many more to come - we need new strategies to establish their efficacy and cost-effectiveness. Conducting the requisite number of phase 3 trials to prove their superiority in terms of their statistical and clinical significance will be difficult. From previous clinical trials of RCC, it is known that phase 2 clinical trials that show higher response rates do not always translate into survival benefits for subjects in the phase 3 trial. It is also known that, despite progression-free survival being the chosen end point in recent phase 3 trials, overall survival is still the gold standard from the clinician's point of view. With the overall survival advantage of the newer systemic therapies, future phase 3 clinical trials will have to deal with the off-study crossover to second- and third-line agents. This tendency will make interpretation of the overall survival data and the ability to decipher which drugs have a true survival benefit more difficult. RCC is not the only disease site to have new-targeted therapies, so we may learn from experience with other tumours. The roles of sequential, combination, neoadjuvant and adjuvant therapy, all need to be tested. The relatively limited number of cases of the disease will make this difficult, but the cost of these agents requires evidence-based prescribing.

As for second- and third-line therapy, who would ever have imagined, even 5 years ago, that a patient with metastatic RCC might have 3 or 4 lines of systemic therapy? Many questions remain. For example, what is the effectiveness of immunother- 
apy after failure of targeted therapy? Is sorafenib clinically beneficial for patients whose treatment with sunitinib failed, or vice versa?

The answers to key questions about the efficacy of second- and third-line therapy depend on understanding the mechanisms of resistance. These answers are likely different for a patient who never responded to therapy (primary resistance) than those for a patient who responded and whose disease progressed some months later (secondary resistance). For patients with primary resistance to therapy, the intuitive conclusion is that the targeted pathway is not vital to the growth of that particular cancer. For patients with secondary resistance, it is possible that the cancer simply bypassed the targeted pathway with a new or upregulated pathway. The optimal strategy for patients with secondary resistance, then, may be offering combination therapy rather than simply switching therapies, as is done for those with primary resistance.

It may be worthwhile to look to our colleagues who treat gastrointestinal stromal tumours for answers. The example of the failure of treatment with imatinib mesylate (Gleevec) is instructive. ${ }^{4,5}$ The rapid clinical decline of patients after discontinuation of the therapy suggested that vital pathways were still being influenced. Significant basic research is being done to better elucidate the mechanisms of resistance, which then must be explored in the clinical setting.

Much is made of the direct and societal costs of the new therapies for RCC. Anyone who prescribes these agents knows that the direct costs of these drugs are significant. The approval process involves a number of steps. For example, sunitinib and sorafenib were both approved by Health Canada in the summer of 2006. However, because they are oral drugs, they must be reviewed at the Common Drug Review (CDR). The CDR reviews not only the therapeutic benefit of a given drug, but also its cost-effectiveness, and provides recommendations to participating publicly funded federal, provincial (except Quebec), and territorial benefit plans in Canada. In February 2007, the CDR announced that it would not approve the use of sorafenib for metastatic RCC. As we write this summary, the decision on sunitinib is still pending. Private insurers may choose to cover these drugs; however, coverage will certainly not be universal.
High drug costs also highlight the differences in reimbursement for oncology-related drugs across the country. For example, in the Western provinces of British Columbia, Alberta and Saskatchewan, the province covers all anticancer drugs, whether they are intravenous, subcutaneous or oral. For the most part, in Atlantic Canada, the province pays for only intravenous drugs. The provinces have traditionally paid for intravenous anticancer medications of proven benefit. Benefit has been defined in different ways, depending on the clinical situation, but has included overall survival, progression-free survival or quality of life benefits. For example, mitoxantrone for hormone-refractory prostate cancer has been approved only on the basis of its improvement of quality of life.

Changes in reimbursement began in 2006. For metastatic colorectal cancer, published data ${ }^{6}$ showed that the addition of bevacizumab (a monoclonal antibody to VEGF) to standard chemotherapy provided a survival advantage of 4.7 months over that with chemotherapy alone. This very quickly became the standard of care in the United States. In Canada, the approval took longer, but Health Canada eventually approved bevacizumab in September 2005. It was then up to each province to review the data and decide whether it would fund the drug through the public healthcare system. Provinces looking at the exact same data arrived at different conclusions. For example, a patient with metastatic colorectal cancer in British Columbia or Newfoundland can receive publicly funded bevacizumab. In other provinces however, the public healthcare system does not pay for this drug; in fact, some regions will not allow a patient to receive the drug in a publicly funded healthcare facility, even if the patient pays for the drug. Similar scenarios with the oral targeted agents for RCC are all too imaginable.

There is no question that this issue will provoke ethical debate. How can Canadians justify a patient being funded to receive sunitinib in Nova Scotia, but not in New Brunswick? Can we accept that patients' home addresses may determine their eligibility to receive effective cancer therapy? This is obviously a very complex issue with many stakeholders, one that may well become a problem for patients with renal cancer and for those treating them over the coming months. A new national process, the Joint Oncology Drug Review, with representation from each province, is being 
piloted. This body will review the efficacy of oncology drugs at a national rather than a provincial level to develop uniformity and consensus and, we hope, to eliminate differences in provincial practice.

Despite concerns about the availability of these new drugs, these are exciting and evolving times in the field of RCC. We continue to be optimistic about and committed to our current and future patients with RCC through our engagement in promising experimental studies and exploration of new therapeutic opportunities.

Competing interests: None declared for Dr. Wood. Dr. Jewett has received an honorarium for being part of an advisory board from Wyeth, Pfizer, Bayer,Biventia Biotech and Paladin Labs.

\section{References}

1. Robson CJ, Churchill BM, Anderson W. The results of radical nephrectomy for renal cell carcinoma. J Urol 1969;101:297-301.

2. Clayman RV, Kavoussi LR, Soper NJ, et al. Laparoscopic nephrectomy: initial case report. J Urol 1991;146:278-82.

3. Novick AC. Laparoscopic and partial nephrectomy. Clin Cancer Res 2004; 10:6322S-7S.

4. Heinrich MC, Corless CL, Blanke CD. Molecular correlates of imatinib resistance in gastrointestinal stromal tumors. J Clin Oncol 2006;24:4764-74.

5. von Mehren M. Imatinib-refractory gastrointestinal stromal tumors: the clinical problem and therapeutic strategies. Curr Oncol Rep 2006;8:192-7.

6. Hurwitz H, Fehrenbacher L, Novotny W. Bevacizumab plus irinotecan, fluorouracil, and leucovorin formetastatic colorectal cancer. N Engl J Med 2004;350:2335-42.

Correspondence: Dr. Lori Wood, Division of Medical Oncology, QEll Health Sciences Centre, Rm. 471, 1278 Tower Rd., Halifax, NS B3H 2Y9; Lori.Wood@cdha nshealth.ca 